\title{
Efeito do contato do sangue e soro fisiológico na alteração de volume e solubilidade dos cimentos de silicato de cálcio MTA HP Repair ${ }^{\circledR}$, Bio-C Repair ${ }^{\circledR}$, MTA Flow ${ }^{\circledR}$ e Bio-C Repair ÍON+®
}

Effect of blood contact and saline solution on volume change and solubility of MTA HP Repairß, Bio-C Repair ${ }^{\circledR}$, MTA Flow ${ }^{\circledR}$ and Bio-C Repair $+\circledR$

Efecto del contacto con la sangre y la solución salina sobre el cambio de volumen y la solubilidad de MTA HP Repair ${ }^{\circledR}$, Bio-C Repair ${ }^{\circledR}$, MTA Flow ${ }^{\circledR}$ y Bio-C Repair ION $+{ }^{\circledR}$

Carlos Alberto Herrero de Morais ORCID: https://orcid.org/0000-0002-5856-5608

Universidade Estadual de Maringá, Brasil E-mail: cahmorais@uem.br

Lyz Cristina Furquim Canali ORCID: https://orcid.org/0000-0002-5886-6767

Universidade de São Paulo, Brasil E-mail: lyzfurquim@usp.br Jussaro Alves Duque ORCID: https://orcid.org/0000-0002-5667-7278 Universidade de São Paulo, Brasil E-mail: jussaroduque@usp.br Murilo Priori Alcalde ORCID: https://orcid.org/0000-0001-8735-065X Universidade de São Paulo, Brasil E-mail: murilo_alcalde@hotmail.com Rodrigo Ricci Vivan ORCID: https://orcid.org/0000-0002-0419-5699 Universidade de São Paulo, Brasil E-mail:rodrigo.vivan@fob.usp.br

Marco Antonio Hungaro Duarte ORCID: https://orcid.org/0000-0003-3051-737X Universidade de São Paulo, Brasil E-mail:mhungaro@fob.usp.br

\begin{abstract}
Resumo
Introdução: O objetivo do presente estudo foi avaliar o efeito do contato do sangue e do soro fisiológico na alteração de volume e solubilidade dos cimentos de silicato de cálcio MTA HP Repair ${ }^{\circledR}$, Bio-C Repair ${ }^{\circledR}$, MTA Flow ${ }^{\circledR}$ e Bio-C Repair $\mathrm{ION}^{+}$( ) Métodos: Após a inserção dos materiais nas cavidades retrógradas, foram escaneados em microtomógrafo 1174 (T0) e em seguida colocados em contato com sangue e soro fisiológico. E depois de 07 dias do contato, os espécimes foram novamente escaneados (T01) e, então, a alteração volumétrica analisada. Os dados foram submetidos à análise estatística pelos testes de WILCOXON, MAN WHITENEY e KRUSKAL-WALLIS ( $\mathrm{p}<0.05)$. Resultados: O contato do soro fisiológico com o cimento Bio-C Repair ÍON ${ }^{+}$promoveu um aumento do seu volume. O sangue proporcionou um aumento da solubilidade do MTA HP e do MTA Flow. Os cimentos Bio-C Repair e Bio-C Repair ÍON $^{+}$não apresentaram alterações de volume quando em contato com sangue. Conclusão: Menor alteração volumétrica quando em contato com sangue ocorreram nos cimentos reparadores pré-misturados.

Palavras-chave: Silicato de cálcio; Solubilidade; Endodontia; Microtomografia computadorizada; Propriedades físico-químicas.

Abstract

The aim of the present study was to evaluate the effect of contact with blood and saline on the change in volume and solubility of calcium silicate cements MTA HP Repair ${ }^{\circledR}$, Bio-C Repair ${ }^{\circledR}$, MTA Flow ${ }^{\circledR}$ and Bio-C Repair ION ${ }^{+\circledR}$. After inserting the materials into retrograde cavities, they were scanned using a microtomograph 1174 (T0), and then placed in contact with blood and saline. After 07 days of contact, the specimens were scanned again (T01), and the volume change and was analyzed. The data were submitted to statistical analysis using the WILCOXON, MAN WHITNEY and KRUSKAL-WALLIS tests $(\mathrm{p}<0.05)$. The contact of saline with Bio-C Repair $\mathrm{ION}^{+}$cement increased its volume.
\end{abstract}


The blood produced an increase in the solubility of MTA HP and MTA Flow. The Bio-C Repair and Bio-C Repair $\mathrm{ION}^{+}$cements did not undergo change in volume when in contact with blood. Lower volume change values occurred in pre-mixed repair cements when they came into contact with blood.

Keywords: Calcium silicate; Solubility; Surgical endodontics; Micro-computed tomography; Physicochemical properties.

\section{Resumen}

El objetivo de este estudio fue evaluar el efecto del contacto con sangre y solución salina sobre el cambio en el volumen y la solubilidad de los cementos de silicato de calcio MTA HP Repair ${ }^{\circledR}$, Bio-C Repair ${ }^{\circ}$, MTA Flow ${ }^{\circledR}$ y

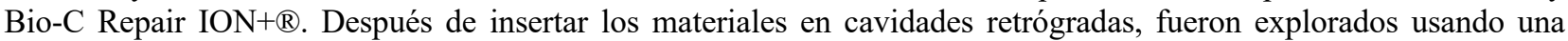
microtomografía 1174 (T0) y después colocados en contacto con sangre y salino. Después de 07 días de contacto, las muestras fueron escaneadas de nuevo (T01), y el cambio de volumen fue analizado. Los datos fueron sometidos a análisis estadístico mediante las pruebas de Wilcoxon, MAN WHITNEY y KRUSKAL-WALLIS $(\mathrm{p}<0,05)$. El contacto de solución salina con cemento Bio-C Repair ION+ aumentó su volumen. La sangre produjo un aumento en la solubilidad de MTA HP y MTA Flow. Los cementos Bio-C Repair y Bio-C Repair ION+ no cambiaron volumen cuando esron en contacto con la sangre. Los valores de menor variación de volumen ocurrieron en los cementos Bio-C Repair y Bio-C Repair ION+ cuando entraron en contacto con la sangre.

Palabras clave: Silicato de cálcio; Solubilidade; Endodoncia quirúrgica; Tomografía micro-computarizada; Propiedades fisicoquímicas.

\section{Introdução}

$\mathrm{Na}$ Endodontia, entre outras aplicações clínicas, o MTA é o material indicado como reparador em perfurações e em obturações retrógradas (Torabinejad \& Chivian, 1999). A capacidade osteogênica, biocompatibilidade, indutora de tecido duro e ação antimicrobiana proporcionada pelo MTA são em função da sua alta alcalinidade corroborando com a reparação e maior previsibilidade de sucesso nos procedimentos (Torabinejad et al., 1995 e 1997; Koh,1997; Holland \& Nery, 2002, Morais et al., 2006; Parirokh \& Torabinejad, 2010).

Apesar de apresentar propriedades físico-químicas e biológicas favoráveis, o MTA é de difícil manuseio, apresenta tempo de presa lento e com potencial para descoloração dentária comprometendo a estética de dentes anteriores (Beloprov \& Parashos, 2011; Duarte et al., 2012). Logo, tem se buscado novas alternativas para suprir essas deficiências.

Com o intuito de melhorar as deficiências do MTA, outros cimentos de silicato de cálcio foram desenvolvidos. Eles são compostos, basicamente, por silicato de cálcio e fosfato de cálcio puro. O radiopacificador pode ser óxido de zircônio ou óxido de tântalo. Esses elementos compõe a primeira fase do material. A segunda fase é composta de fosfato monobásico de cálcio que, após hidratação, promove a liberação de íons cálcio que juntamente com os íons fosfato proveniente dos tecidos iniciam e promovem a biomineralização. Segundo os fabricantes, esses cimentos apresentam como principais vantagens suas propriedades físico-químicas e biológicas como: $\mathrm{pH}$ alcalino, atividade antibacteriana, radiopacidade, biocompatibilidade, não toxicidade, quimicamente estável em ambiente biológico, além de induzirem a formação de hidroxidoapatita e promoverem uma ligação entre dentina e material obturador realizando um bom selamento (Camilleri, 2008; Loushine et al., 2011; Candeiro, et al., 2016 ; Kohli et al., 2015; Camilleri, 2017 ; Cavenago et al., 2017; Cintra et al., 2017; Marciano et al., 2017; Silva et al., 2017 ; Sisli et al., 2017).

Nekoofar et al. (2010) investigaram os efeitos da contaminação por sangue sobre a resistência à força de compressão e microestrutura de superfície do MTA (Pro-Root ${ }^{\circledR}$ ). Observaram que quanto mais o MTA foi incorporado por sangue, menor foi a sua resistência à compressão. E, em nível de microestrutura, a contaminação do MTA por sangue resultou na ausência de cristais aciculares, o que promoveu a redução na resistência à força de compressão.

Ainda, Nekoofar et al. (2010) ao avaliarem o efeito do sangue e do soro fisiológico sobre a microdureza e microestrutura de superfície do MTA branco e cinza a curto e a longo prazo, verificaram que, o MTA branco teve uma maior microdureza de superfície que o MTA cinza. Além disso, concluíram que a contaminação por sangue resultou e um efeito 
prejudicial sobre a microdureza e microestrutura de superfície do MTA. Logo, sugeriram que devem ser realizadas tentativas para controlar o sangramento nas aplicações clínicas.

O MTA-HP Repair®, já considerado um cimento biocerâmico, devido à melhoria das suas propriedades físicoquímicas, apresenta a adição de um plastificante ao líquido, o radiopacificador é o tungstato de cálcio e diminuiu-se o tamanho das partículas. O MTA Flow ${ }^{\circledR}$ também consiste de um cimento com veículo viscoso e partículas muito finas o que lhe confere grande fluidez.

Na mesma linha de cimentos de silicato de cálcio, porém, dispensando a manipulação, composto por nanopartículas, acondicionados em seringas e prontos para uso, a Angelus lançou 2019 o Bio-C Repair® no Brasil e em 2020 o Bio-C Repair $\mathrm{ION}^{+} \AA$ nos USA. Segundo o fabricante, o ternário que dá origem ao cimento Bio-C Repair ${ }^{\circledR}$ é composto basicamente por óxido de silício, óxido de alumínio e óxido de cálcio. O Bio-C Repair ÍON ${ }^{+} \circledast$, é um cimento de silicato de cálcio modificado, aonde se substituiu, no ternário que lhe deu origem, a substituição do óxido de alumínio pelo óxido de magnésio.

Como a contaminação do material por sangue é inevitável e uma situação clínica comum nas cirurgias parendodônticas e selamento de perfurações. Há poucos estudos na literatura que analisaram o efeito do sangue nos novos cimentos de silicato de cálcio, principalmente nos pré-misturados. Segundo Camilleri (2020), podem ocorrer alterações da estrutura e superfície química dos cimentos hidráulicos quando do contato com sangue. Esta alteração deve ser investigada, pois podem trazer efeitos deletérios ao material, como na degradação e aumento da solubilidade.

O objetivo deste estudo foi investigar, utilizando-se da pesquisa laboratorial, quantitativa, a interferência do sangue e soro fisiológico na alteração de volume e solubilidade do MTA Repair HP®, MTA Flow ${ }^{\circledR}$, Bio-C Repair ${ }^{\circledR}$ e Bio-C Repair ÍON$^{+}$(. Para isso utilizou-se da microtomografia computadorizada. A hipótese nula é que sangue e soro físiológico não interfiram na alteração de volume dos cimentos de silicato de cálcio.

\section{Metodologia}

O protocolo desse estudo foi recebido e aprovado pelo comitê de ética da Universidade Estadual de Maringá (CAAE: 90034318.6.0000.0104).

Material

Os materiais utilizados para este estudo foram MTA Repair HP® (Angelus, Londrina, PR, Brasil) com lote $\mathrm{n}^{\circ} 42683$, MTA Flow ${ }^{\circledR}$ (Ultradent Products Inc., South Jordan, UT, USA) com lote $n^{\circ}$ 2019032703, Bio-C Repair ${ }^{\circledR}$ (Angelus, Londrina, PR, Brasil) com lote ${ }^{\circ} 46498$ e Bio-C Repair ÍON ${ }^{+}$(Angelus, Londrina, PR, Brasil) com lote ${ }^{\circ} 150519$.

Preparo dos espécimes

Os espécimes foram confeccionados utilizando-se de um incisivo central superior permanente de humano com apicetomia de $3 \mathrm{~mm}$ apicais e cavidade retrógrada com $3 \mathrm{~mm}$ de profundidade realizada com broca carbide $\mathrm{n}^{\circ} 2$ (Beavers Dental, Morrisburg, ON, Canadá) em alta rotação com abundante refrigeração. Após a moldagem com silicone de adição (Zhermak, Badia Polesine, Italy) e obtenção do molde foram reproduzidos 80 réplicas da raiz do dente em resina acrílica (Clássico, Campo Limpo Paulista, SP, Brasil). Em todos os espécimes, utilizando-se de uma régua endodôntica milimetrada e broca carbide esférica $\mathrm{n}^{\circ} 2$, foi realizado a confirmação da profundidade e possíveis ajustes quando necessário para padronizar todas as cavidades retrógradas, utilizando-se de um microscópio operatório (DF Vasconcelos, Rio de Janeiro, Brasil) com 20X de aumento (Figura 1). 
Figura 1 - Preparo dos espécimes
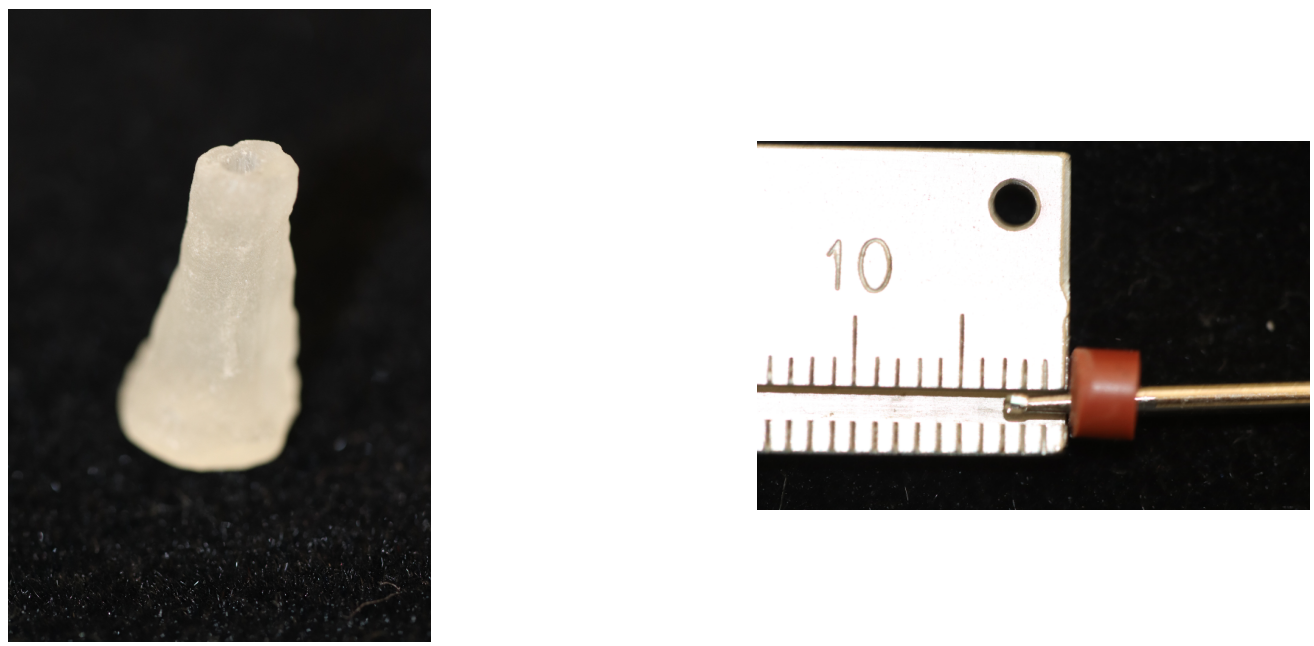

Espécime com cavidade retrógrada preparada com broca esférica $\mathrm{n}^{\circ} 2 \mathrm{com} 3 \mathrm{~mm}$ de profundidade. Fonte: Autores.

Os espécimes foram divididos em 8 grupos experimentais, conforme a substância a que foram colocados em contato, soro fisiológico ou sangue (Tabela1).

Tabela 1 - Distribuição dos grupos experimentais com relação aos materiais utilizados e as substâncias em contato

\begin{tabular}{c|c|c|c}
\hline Grupos & Material & Contato com substância & Espécimes \\
1 & MTA Repair HP & soro fisiológico & 10 \\
2 & MTA Repair HP & sangue & 10 \\
3 & Bio-C Repair & soro fisiológico & 10 \\
4 & Bio-C Repair & sangue & 10 \\
5 & MTA Flow & soro fisiológico & 10 \\
6 & MTA Flow & sangue & 10 \\
7 & Bio-C Repair ÍON & 10 \\
8 & Bio-C Repair ÍON & soro fisiológico & 10 \\
\hline
\end{tabular}

Fonte: Autores.

Após o preparo do material, as cavidades retrógradas dos espécimes foram preenchidas utilizando-se de porta MTA, espátula de inserção e calcadores endodônticos (Figura 2). 
Figura 2 - Preenchimento da cavidade retrógrada.

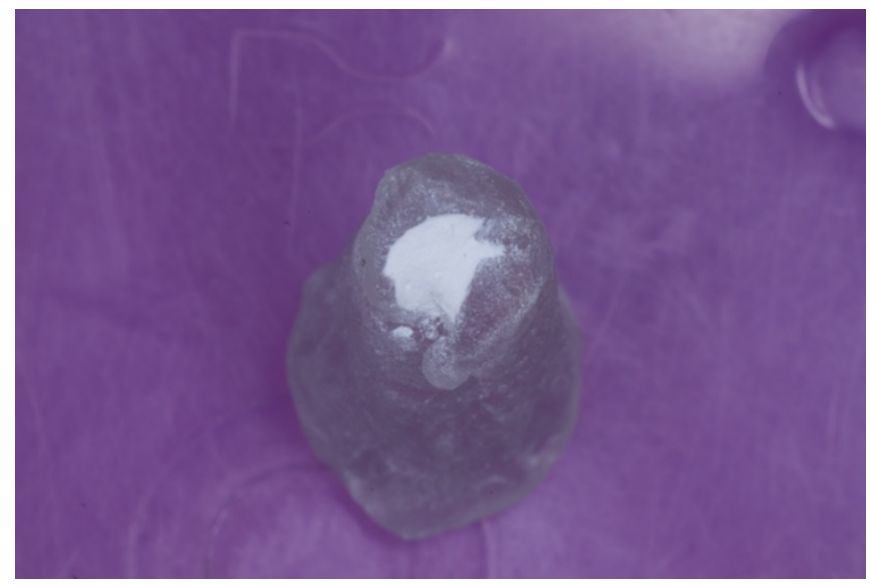

Espécime com cavidade retrógrada preenchida com cimento de silicato de cálcio. Fonte: Autores.

Os espécimes foram inspecionados, novamente, e visualmente com o auxílio do microscópio operatório (DF Vasconcelos ${ }^{\circledR}$, Valença, RJ, Brasil) com 20X de aumento para garantir que não houvessem espaços vazios ou bolhas. Assim, os espécimes que apresentaram falhas, bolhas ou espaços vazios foram excluídos desse estudo.

Depois de preenchidos e inspecionados, os espécimes com obturação retrógrada foram distribuídos em eppendorfs e divididos em 08 grupos de 10 espécimes cada (80 amostras), conforme Tabela 1. Foram preparados os microtubos tipo eppendorf (Cralplast ${ }^{\circledR}$, Artigos para Laboratório LTDA, Cotia, SP, Brasil) com a inserção de uma esponja hemostática de colágeno (Hemospon ${ }^{\circledR}$, Maquira Indústria de Produtos Odontológicos S.A., Maringá, PR, Brasil) para simular uma situação clínica como os tecidos peripaicais (Figura 3).

Figura 3 - Preenchimento do microtubo.

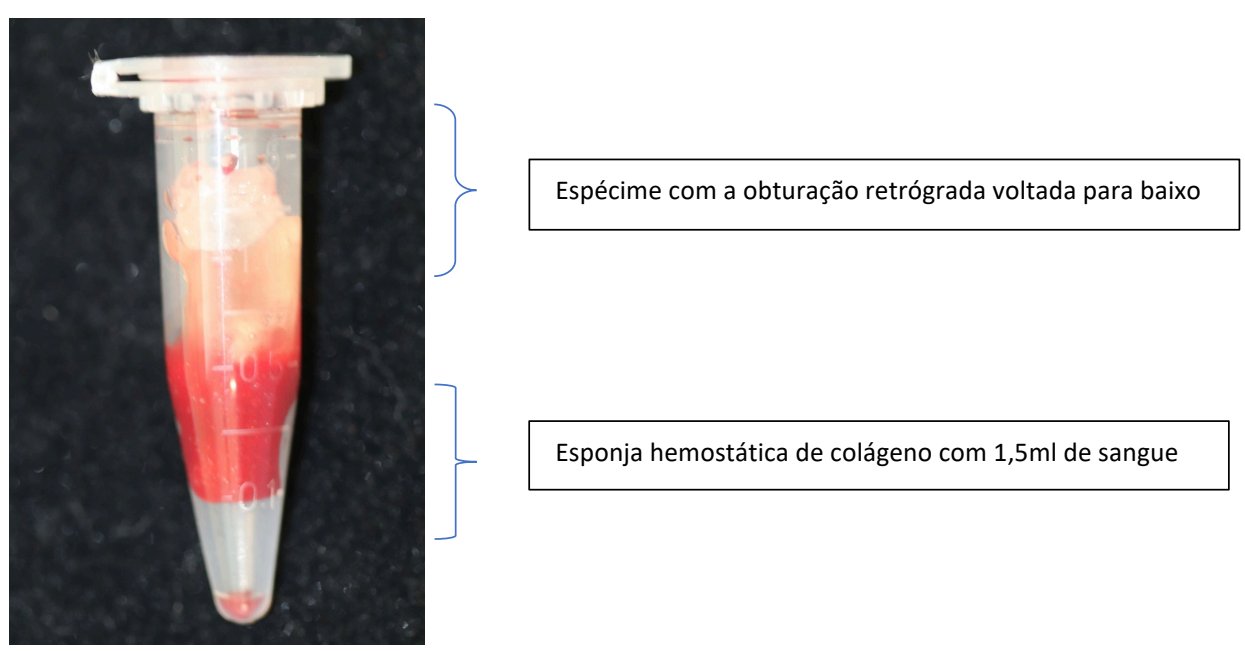

Espécime dentro do microtubo com esponja de colágeno e sangue. Fonte: Autores.

Em todos os grupos foram adicionados em cada eppendorf 1,5 $\mathrm{mL}$ de soro físiológico ou sangue de carneiro desfibrinado (Laborclin ${ }^{\circledR}$, Pinhais, PR, Brasil). 


\section{Alteração de volume e solubilidade}

Após a inserção do material nas cavidades retrógradas os espécimes foram escaneados no microtomógrafo imediatamente com o tempo $\mathrm{T} 0$ e em seguida foram acondicionados nos eppendorfs com a obturação retrógrada em contato com a esponja de fibrina umedecida com soro fisiológico ou sangue (Figura 3).

O scanner utilizado foi de raios-X microfocus CT (1174v2 SkyScan; SkyScan, Kontich, Bélgica), aonde se escaneava uma amostra de cada vez. A alteração de volume e solubilidade foi avaliada pelas medições volumétricas dos cimentos por meio de imagens Micro-CT, a área de trabalho de cada um dos grupos, sendo a obturação retrógrada usada para o cálculo do volume inicial de cada amostra.

O procedimento de digitalização foi completado utilizando $50 \mathrm{KV}$ de tensões de raios-X e $800 \mu \mathrm{A}$ de corrente de ânodo. Os parâmetros de captura de imagem utilizados foram de um tamanho de $14.1 \mathrm{~lm}$ voxel com $1,1^{\circ}$ passo de rotação, usada uma rotação de $360^{\circ}$. Cada varredura consistiu em imagens 327. tif com 1.02491 .304 pixels. Os dados digitais foram ainda elaborados pelo software de reconstrução (NREConv 1. 6.4.8, SkyScan) e o software CTAN (v1.11.10.0 CTAN, SkyScan) e foi utilizado para as medições de volume. No software CTAN, as amostras presentes na imagem foram separadas para serem analisadas individualmente. O software se limitou a analisar a área de interesse (ROI) individualmente para cada uma das amostras e os novos dados de ROI foram salvos em pastas separadas. Em seguida, o conjunto de dados obtidos das amostras foi aberto e o valor do binário foi ajustado de acordo com as imagens. Este valor foi registrado para ser usado mais tarde na segunda digitalização. Através da reprodução das imagens em 3D foi possível obter a análise quantitativa do volume inicial de material. Desta forma, isso promoveu uma ferramenta automatizada de cálculo do volume total (3 mm) a partir da imagem tridimensional (3D) dos objetos selecionados binários (de cor branca). Depois de executar a função de análise de '3D', estes dados foram registrados em uma lista de resultados.

Os grupos foram armazenados em estufa com temperatura ambiente $25^{\circ} \mathrm{C}$, por 07 dias.

\section{Análise estatística}

A análise da solubilidade dos materiais intra-grupos, intra-materiais e entre grupos foram comparadas respectivamente pelos testes de WILCOXON, MAN WHITENEY E KRUSKAL-WALLIS $(\mathrm{p}<0.05)$. Foi utilizado o software Prism 5.0 (GraphPad Software Inc, La Jolla, CA, EUA) como ferramenta analítica.

\section{Resultados e Discussão}

Na comparação entre os volumes inicial (T0) e após 07 dias dentro de um mesmo grupo (T01), só foi observada diferença significante $(\mathrm{P}<0.05)$ no grupo do Bio-C Repair ÍON $^{+}$em contato com soro fisiológico, onde ocorreu um aumento de volume significante após 07 dias. Nos demais grupos não houveram diferenças significantes nas comparações entre o período inicial (T0) e após 07 dias ( $\mathrm{P}>0.05)$ (Tabela 2).

Quando da análise da porcentagem de solubilidade, na comparação entre os grupos em contato com soro fisiológico não houveram diferenças significantes $(\mathrm{P}>0.05)$. Entretanto, em contato com sangue houveram diferenças significantes quando se comparou o grupo do Bio-C Repair ÍON ${ }^{+}$e o grupo do Bio-C Repair com o grupo do MTA HP e grupo do MTA Flow $(\mathrm{P}<0.05)$. Nas demais comparações não houveram diferenças significantes $(\mathrm{P}>0.05)$. Na comparação entre meios de contato dentro de um mesmo material, houveram diferenças significantes entre o contato com soro fisiológico em relação ao contato com sangue apenas no grupo do MTA HP e no grupo do MTA Flow $(\mathrm{P}<0.05)$ (Tabela 3). 
Tabela 2 - Mediana (Med), valores mínimos (Min) e máximos (Max) do volume do material imediatamente após inserção do material (T0) e 07 dias após o contato com soro fisiológico ou sangue (T01)

\begin{tabular}{|c|c|c|c|c|}
\hline Grupos & Material & $\begin{array}{l}\text { Contato } \\
\text { com } \\
\text { substância } \\
\end{array}$ & T0 Med (Min/Max) & $\begin{array}{l}\text { T1 Med } \\
\text { (Min/Max) }\end{array}$ \\
\hline 1 & MTA HP & Soro & $\begin{array}{c}1,96^{\mathrm{A}} \\
(1,13-2,91)\end{array}$ & $\begin{array}{c}2,02^{\mathrm{A}} \\
(1,16-2,86)\end{array}$ \\
\hline 2 & MTA HP & Sangue & $\begin{array}{c}1,39^{\mathrm{A}} \\
(1,05-2,43)\end{array}$ & $\begin{array}{c}1,29^{\mathrm{A}} \\
(0,74-2,35)\end{array}$ \\
\hline 3 & Bio C Repair & Soro & $\begin{array}{c}0,88^{\mathrm{A}} \\
(0,55-1,33) \\
\end{array}$ & $\begin{array}{c}0,86^{\mathrm{A}} \\
(0,62-1,59)\end{array}$ \\
\hline 4 & Bio C Repair & Sangue & $\begin{array}{c}1.02^{\mathrm{A}} \\
(0,55-1,33)\end{array}$ & $\begin{array}{c}1,03^{\mathrm{A}} \\
(0,56-1,33)\end{array}$ \\
\hline 5 & MTA Flow & Soro & $\begin{array}{c}1,57^{\mathrm{A}} \\
(1,15-2,06)\end{array}$ & $\begin{array}{c}1,61^{\mathrm{A}} \\
(1,22-2,09)\end{array}$ \\
\hline 6 & MTA Flow & Sangue & $\begin{array}{c}1,39^{\mathrm{A}} \\
(1,05-2,43)\end{array}$ & $\begin{array}{c}1,29^{\mathrm{A}} \\
(0,74-2,35)\end{array}$ \\
\hline 7 & $\begin{array}{l}\text { Bio-C Repair ÍON }{ }^{+} \\
\text {Bio-C Repair ÍON }{ }^{+}\end{array}$ & $\begin{array}{l}\text { Soro } \\
\text { Sangue }\end{array}$ & $\begin{array}{c}0,84^{\mathrm{A}} \\
(0,53-1,69) \\
1,04^{\mathrm{A}} \\
(0,69-1,26)\end{array}$ & $\begin{array}{c}0,85^{\mathrm{B}} \\
(5,34-16,24) \\
1,05^{\mathrm{A}} \\
(0,67-1,26)\end{array}$ \\
\hline
\end{tabular}

Letras diferentes indicam diferenças significantes entre o volume pré e pós de cada material $(\mathrm{P}<0,05)$. Fonte: Autores.

Tabela 3 - Mediana, valores mínimo e máximo da porcentagem de solubilidade entre os materiais estudados em função do meio de contato (Sinal - indica perda volumétrica e sinal + indica aumento de volume).

\begin{tabular}{l|l|l|l|l}
\hline & MTA HP & Bio-C Repair & MTA Flow & $\begin{array}{l}\text { Bio-C Repair } \\
\text { ION }^{+}\end{array}$ \\
\hline Soro & $+0,83^{\mathrm{Aa}}$ & $-0,40^{\mathrm{Aa}}$ & $+2,43^{\mathrm{Aa}}$ & $+1,25^{\mathrm{Aa}}$ \\
& $(-9,74-+3,70)$ & $(-21,33-+8,89)$ & $(-8,65-+17,43)$ & $(-2,28-+7,05$ \\
\hline Sangue & $-8,31^{\mathrm{Ba}}$ & $+0,39^{\mathrm{Ab}}$ & $-13,55^{\mathrm{Ba}}$ & $+0.03^{\mathrm{Ab}}$ \\
& $(-45,49-+45,20)$ & $(-5,38-+3,91)$ & $(-36,21-+47,08)$ & $(-5,92-+2,57)$ \\
\hline
\end{tabular}

Letras maiúsculas diferentes indicam diferenças significantes entre os mesmos materiais em função do meio de imersão $(\mathrm{P}<0,05)$. Letras minúsculas diferentes indicam diferenças os materiais dentro de um mesmo meio de imersão $(\mathrm{P}<0,05)$. Fonte: Autores. 
Figura 4 - Imagens representativas da alteração volumétrica dos materiais após a imersão em solução salina e sangue. A cor vermelha indica o aumento de volume dos materiais, enquanto que a cor amarela indica a diminuição de volume dos materiais.

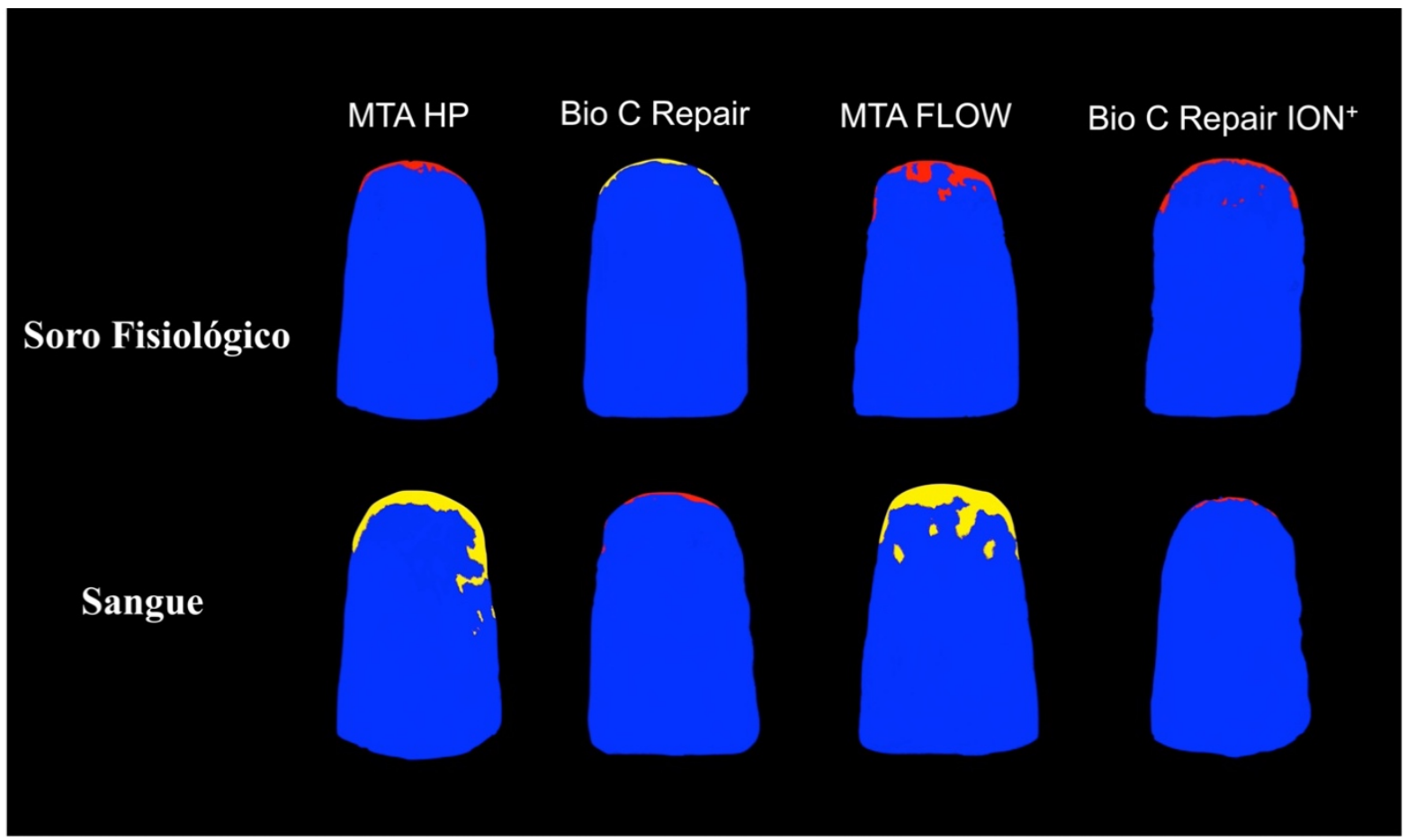

Fonte: Autores.

O presente estudo avaliou a alteração volumétrica dos cimentos de silicato de cálcio MTA HP, Bio C Repair, MTA Flow e Bio C Repair Íon+, em obturações retrógradas, em contato com sangue e soro fisiológico.

A hipótese nula foi rejeitada, visto que, houveram alteração de volume dos cimentos de silicato.

Nesse estudo, o grupo do cimento Bio-C Repair $\mathrm{ION}^{+}$mostrou aumento do volume quando em contato com soro fisiológico. Essa diferença foi estatisticamente significante entre os grupos $(p<0.05)$ (Tabela 2). Segundo o fabricante, no mecanismo de ação Bio-C Repair ÍON ${ }^{+}$, ocorre o imbricamento de cristais em forma de agulhas do sulfato de cálcio dihidradato com os cristais do silicato de cálcio hidratado modificado. Esta reação química proporciona maior coesão e resistência mecânica ao cimento e ao mesmo tempo a interação entre os cristais formados promove uma pequena, mas desejável expansão do cimento. Isso corrobora os nossos resultados. Segundo Siboni e col. (2017), os cimentos de silicato de cálcio são mais hidrofílicos e consequentemente mais solúveis. O cimento Bio-C Repair ÍON ${ }^{+}$apesar de absorver líquido, não apresentou grande solubilidade. Segundo o fabricante, o cimento Bio-C Repair ÍON ${ }^{+}$ao entrar em contato com a água, o sulfato de cálcio hemi-hidratado ( $\mathrm{CaSO} 4.1 / 2 \mathrm{H} 2 \mathrm{O})$ presente na sua composição é dissolvido em dihidratado (CaSO4.2H2O), o qual é pouco solúvel, promovendo a saturação do seu ambiente fisiológico e consequentemente à precipitação em cristais em forma de agulhas oque vem explicar os nossos resultados. Este cimento ainda é recente na Endodontia e outros trabalhos devem avaliar suas propriedades físico-químicas e aplicação nos procedimentos clínicos.

A grande perda volumétrica pode aumentar a possibilidade de infiltração bacteriana e consequentemente levar a falha do procedimento endodôntico (Wu et al.,1995). A solubilidade é a propriedade relatada pela dissociação e degradação dos constituintes do material pela ação de contato com os fluidos teciduais.

Segundo a norma da ISO 6876 recomenda que o material endodôntico deve apresentar solubilidade inferior a 3\%. Porém, segundo Fridland (2003) e Ostarvik (2014), algum grau de solubilidade é necessário para que o material apresente bioatividade. Isto confere aos materiais maior liberação de íons, elevando o $\mathrm{pH}$ e promovendo maior ação antibacteriana, tecidual e celular nos processos de cura e reparo. No nosso estudo o MTA HP quando em contato com sangue, apresentou uma 
solubilidade de $8.31 \%$, bem superior a 3\% da norma ISO. Essa diferença foi estatisticamente significante ( $\mathrm{p}<0.05$ ) (Tabela 3 e Figura 4). Esses resultados corroboram com outro estudo prévio (Guimarães e col., 2018) aonde MTA HP Repair apresentou solubilidade de 8,18\% quando comparado ao MTA convencional. Segundo os autores, (Guimarães e col., 2018) a presença do plastificante no líquido do MTA HP, aumenta a sua solubilidade.

De acordo com Cintra et al. (2017), o MTA HP possui alta plasticidade quando comparado ao MTA convencional. O pó composto por pequenas partículas de silicato dicálcico, silicato tricálcico, tricálcio aluminato, óxido de cálcio e tungstato de cálcio, quando misturados ao líquido com plastificante, é fácil de manipular e promovem uma consistência similar a massa de modelar, não arenosa, de fácil inserção nas cavidades. O MTA Flow apresenta características similares ao MTA HP e segundo Guimarães e col. (2018) a presença de gel agregado com a água no liquido desse cimento, favorece a sua manipulação e inserção nas cavidades.

No nosso estudo o MTA Flow quando em contato com sangue apresentou uma solubilidade de $13.55 \%$. Essa diferença foi estatisticamente significante $(\mathrm{p}<0.05)$ (Tabela 3 e Figura 4). Esse resultado possivelmente se deve a capacidade de reação dos constituintes desses cimentos com sangue aumentando a sua solubilidade. A mesma situação ocorreu com o MTA Flow (Guimarães e col., 2018), também verificaram que o veículo de gel a base de água, facilitou a sua solubilidade. Esses achados corroboraram nossos resultados.

Nesse estudo utilizamos réplicas de dentes em resina acrílica com cavidades retrógradas com $3 \mathrm{~mm}$ de profundidade como Cavenago et al. (2014); Canali e col. (2016) e Guimarães et al. (2018) (Figura 1) e preenchidas com cimento de silicato de cálcio (Figura 2). Quanto as substâncias de contato utilizadas, tanto o soro fisiológico ou sangue, apenas a quantidade adicionada nos eppendorfs e absorvida pela esponja de fibrina serviram de meio de acondicionamento para contato os espécimes, procurando desta forma, estabelecer uma situação mais próxima da realidade clínica. Outros estudos, como Cavenago et al. (2014); Canali e col. (2016) e Guimarães et al. (2018), mantiveram os espécimes imersos em água durante o período de 168 horas ou 07 dias.

Quando comparamos os meios de contato empregados nesse estudo, ou seja, soro fisiológico e sangue, com MTA HP e MTA Flow, o sangue mostrou uma diferença estatisticamente significante em relação ao soro fisiológico ( $<<0.05$ ) (Tabela 3). Houve uma perda de volume de $8.31 \%$ e $13.55 \%$ respectivamente, o mesmo ocorreu no trabalho de Siboni e col. (2017) que verificaram solubilidade maior que $3 \%$ nos cimentos de silicato de cálcio. Isso ocorreu provavelmente devido aos veículos plastificantes e gel desses cimentos, aumentando dessa forma a sua solubilidade. Corroborando nossos resultados, Nekoofar et al. (2010), também observaram que o sangue afetou os materiais estudados e mostrou uma diminuição na força de compressão com o sangue e no EDS uma alteração dos cristais do MTA.

Akinci et al. (2020) verificaram que o contato da obturação retrógrada de cimento de silicato de cálcio (Biodentine®) com sangue promoveu perda de volume e densidade. Relata ainda que a coagulação do sangue nos trabalhos "in vitro" poderia não simular adequadamente as situações clínicas "in vivo". Nekoofar et al. (2010), também mostraram que a adição de anticoagulante ao sangue humano, como o citrato, pode interferir no tempo de presa do MTA. Para evitar tais problemas e simular melhor a situação clínica, no nosso estudo utilizamos o sangue de carneiro desfibrinado, o qual retardou o processo de coagulação. Segundo Slattery \& Beaumont (1989), mostraram a similaridade entre os componentes do sangue de carneiro e sangue humano, e da possiblidade do seu emprego como modelo de pesquisa.

No nosso estudo observamos que os cimentos de silicato de cálcio prontos para uso, Bio-C Repair e Bio-C Repair $\mathrm{I}^{\mathrm{O}} \mathrm{N}^{+}$, não sofreram alteração de volume expressiva, principalmente, quando em contato com sangue. Isso mostra que esses cimentos têm excelente estabilidade dimensional e dessa forma possivelmente não deverá ocorrer a percolação apical da obturação retrógrada. Possivelmente essa maior estabilidade pode estar relacionada a menor dimensão de partícula que confere 
maior homogeneidade ao material e menor risco de degradação. Dessa forma, há de se esperar um prognóstico mais favorável das cirurgias parendodônticas quando da utilização desses materiais.

Esses resultados ainda são motivos de estudos e mostram a importância de se avaliar as alterações promovidas nos materiais na hora da sua utilização, visto que, a presença do sangue é uma condição comum e inevitável nas aplicações clínicas.

\section{Conclusão}

Em suma esse estudo revelou que o contato do cimento Bio-C Repair ÍON ${ }^{+}$com soro fisiológico promoveu aumento do seu volume.

O contato do sangue com MTA HP e MTA Flow promoveu aumento da solubilidade.

Os cimentos Bio-C Repair e Bio-C Repair ÍON ${ }^{+}$não apresentaram aumento da solubilidade em contato com ambas substâncias.

Ainda é motivo de estudo as possíveis alterações nas propriedades físicas nos cimentos de silicato de cálcio quando em contato com sangue.

\section{Referências}

Akinci L., Simsek N., \& Aydinbelge H. A. (2020). Physical properties of MTA, BioAggegate and Biodentine in simulated conditions: A micro-CT analysis. Dental materials journal, $1-7$.

Belobrov I. \& Parashos P. (2011). Treatment of tooth discoloration after the use of white mineral trioxide aggregate. Journal of endodontics., 37 (7), 10171020 .

Camilleri J. (2008). Characterization of hydration products of mineral trioxide aggregate. International endodontic journal, $41,408-417$.

Camilleri J. (2017). Will bioceramics be the Future Root Canal Filling Materials? Dental restorative materials, Curr oral health rep.

Camilleri J. (2020). Classification of hydraulic cements used in dentistry. Frontiers in dental medicine, 1, (9), 1-6.

Candeiro G. T., Moura-Netto C., D’Almeida-Couto R. S., Azambuja-Junior, N., Marques M. M., Cai S., \& Gavini G. (2016) Cytotoxity, genotoxicity and antibacterial effectiviness of bioceramic endodontic sealer. International endodontic journal, 49, 858-864.

Canali L. C. F., Morais C. A. H., Cavenago B. C., Vivan R. R., \& Duarte M. A. H. (2016). Influência do contato com sangue e soro fisiológico na solubilidade, pH e constituição química do MTA. Dental press endodontics, 6, (3), 41- 45.

Cavenago B. C., Pereira T. C., Duarte M. A. H., Ordinola-Zapata R., Marciano M. A., Bramante C. M., \& Bernardineli N. (2014). Influence of powder-towater ratio on radiopacity, setting time, $\mathrm{pH}$, calcium release and micro-CT volumetric solubility of white mineral trioxide aggregate. International endodontic journal, 47, 120-126.

Cavenago B. C., Carpio-Perochena A. E. D., Ordinola-Zapata R., Estela C., Garlet G. P., Tanomaru-Filho M., Weckwerth P. H., Andrade F. B., \& Duarte M. A. H. (2017). Effect of using different vehicles on the physicochemical, antimicrobial, and biological properties of mineral trioxide aggregate. Journal of endodontics, 43, 779-786.

Cintra, L. T. A., Benetti F., Queiroz I. O. A., Lopes J. M. A., Oliveira S. H. P., Araújo G. S., \& Gomes-Filho J. E. (2017). Cytotoxicity, biocompatibility, and biomineralization of the new high-plasticity MTA material. Journal of endodontics, 43, 774-778.

Hungaro Duarte M. A., Minotti P. G., Rodrigues C. T. et al. (2012). Effect of different radiopacifying agents on the physicochemical properties of white Portland cement and white mineral trioxide aggregate. Journal of endodontics, 38, (3), 394-397.

Fridland M. \& Rosado R. (2003). Mineral trioxide aggregate (MTA) solubility and porosity with different water-to-powder ratios. Journal of endodontics, 29, $814-817$.

Guimarães B. M., Prati C., Duarte M. A. H., Bramante C. M., \& Gandolfi M. G. (2018). Physicochemical properties of calcium silicate-based formulations MTA Repair HP and MTA Vitalcem. Jaos, 26, 1-8.

Holland R., de Souza V., Nery M. J. et al. (2002). Calcium salts deposition in rat connective tissue after the implantation of calcium hydroxide-containing sealers. Journal of endodontics, 28,173-176.

International Organization for Standardization (2001). Dental root canal sealing materials, ISO 6786.

Koh E. T. et al. (1997). Mineral trioxide aggregate stimulates a biological response in human osteoblasts. Journal of biomed materials research, 37, (3), 432439. 
Kohli M. R., Yamaguchi M., Setzer F. C., \& Karabucak B. (2015). Spectrophotometric analysis of coronal tooth discolorations induced by various bioceramic cements e other endodontic material. Journal of endodontics, 41, 1862-1866, 2015.

Loushine, B. A., Bryan T. E., \& Looney S. W. (2011). Setting properties and cytotoxicity evaluation of premixed bioceramic root canal sealer. Journal of endodontics, 37, 673-677.

Marciano M. A., Camillieri J., Costa R. M., Matsumoto M. A., Guimarães B. M., \& Duarte M. A. H. (2017). Zinc oxide inhibits dental discoloration caused by white mineral aggregate angelus. Journal of endodontics, 43, 1001-1007.

Morais C. A. H. et al. Evaluation of tissue response to MTA and Portland cement with iodoform. (2006). Oral surgery oral medicine oral pathology oral radiology endodontics, 102, 417-421.

Nekoofar M. H., Aseeley Z., \& Dummer P. M. (2010). The effect of various mixing techniques on the surface microhardness of mineral trioxide aggregate. International endodontic journal, 43, 312-320.

Nekoofar M. H., Oloomi K., Sheykhrezae M. S., Tabor R., Stone D. F., \& Dummer P. M. H. (2010). An evaluation of the effect of blood and human serum on the surface microhardness and surface microstructure of mineral trioxide aggregate. International endodontic journal, 43, 849-858.

Orstavik D. (2014). Endodontic filling materials. Endodontic topics. 31, 53-67.

Parirokh M. \& Torabinejad M. (2010). Mineral trioxide aggregate: a comprehensive literature review-Part I: chemical, physical, and antibacterial properties. Journal of endodontics, 36, 16-27.

Siboni F., Taddei P., Zamparini F., Prati C., \& Gandolfi M. G. (2017). Properties of BioRoot RCS, a tricalcium silicate endodontic sealer modified with povidone and polycarboxylate. International endodontic journal, 50, 120-136.

Silva E. J. N. L., Carvalho N. K., Guberman M. R. C., Pardo M., Senna P. M., Souza E. M., \& De-Deus G. (2017). Push out bond strength of fast-setting mineral trioxide aggregate and pozzolan-based cements: Endocem MTA and Endocem Zr. Journal of endodontics, 43, 801-804.

Sisli S. N. \& Ozbas H. (2017). Comparative micro-computed tomographic evaluation of the sealing quality of Pro Root MTA and MTA Angelus apical plugs placed with various techniques. Journal of endodontics, 43, 147-151.

Slattery C. \& Beaumont D. (1989). Sheep platelets as a model for human platelets: Evidence for specific PAF (Platelet Activating Factor) receptors. Thrombosis research, 55,569-576.

Torabinejad M., Hong C. U., McDonald F., \& Pitt Ford T. R. (1995). Physical and chemical properties of a new root-end filling material. Journal of endodontics, 21, 349-353.

Torabinejad M., Hong C. U., Pitt Ford T. R., \& Kaiyawasam S. P. (1995). Tissue reaction to implanted super-EBA and mineral trioxide aggregate in the mandible of guinea pigs: a preliminary report. Journal of endodontics, 21, 569-571.

Torabinejad M., Pitt Ford T. R., McKendry D. J., Abedi H. R., Miller D. A., \& Kariyawasam S. P. (1997). Histologic assessment of mineral trioxide aggregate as a root-end filling in monkeys. Journal of endodontics, 23, 225-228.

Torabinejad M. \& Chivian N. (1999). Clinical applications of mineral trioxide aggregate. Journal of endodontics, 25, (3),197-205.

Torabinejad M et al. (1995). Investigation of mineral trioxide aggregate for root-end filling in dogs. Journal of endodontics, 21 , (12), $603-607$.

Wu M. K. et al. (1995). A 1-year follow-up study on leakage of four root canal sealers at different thicknesses. International endodontics journal., 28,185-189. 